\title{
GENERALIZED MEANS OF SUBHARMONIC FUNCTIONS
}

\section{S. J. GARDINER}

\section{Introduction}

This paper is concerned with means of subharmonic functions over various bounded surfaces in Euclidean space $\mathbf{R}^{n}(n \geqq 2)$. The simplest case is that of spherical means, which have played a fundamental rôle in the development of potential theory ever since the pioneering work of F. Riesz [26] in 1926. In particular, they have convexity properties, and their limiting behaviour for large radii may be used as a criterion for (e.g.) harmonic majorization in $\mathbf{R}^{n}$. A number of such properties are listed below in Theorem 11 (Section 12). However, if we wish to deal with a subharmonic function defined only in an unbounded proper subdomain of $\mathbf{R}^{n}$, then means over spheres with a common centre and arbitrarily large radii can no longer be considered.

In the half-space this problem was overcome by devising a "weighted" halfspherical mean, the development of which can be traced through papers by Ahlfors [1], Tsuji [27], Huber [19], Dinghas [11], Ahlfors [2], Kuran [22], [23] and Armitage [3], [4]. A corresponding cylindrical mean in the infinite strip, studied by Heins [17] and Brawn [8], [9], has only recently [5] been explored to an extent that approaches the half-spherical mean, and Fugard [13] has analogously investigated conical means in the infinite cone.

Each of these weighted means has been separately studied at some length, and shown to behave in a manner very similar to spherical means. In this paper we extend the work of [14] and present a unified theory of such means, which we define in terms of level surfaces of suitable functions. Some links may be seen here with work by $\mathrm{Wu}[29]$, who considers integral means of subharmonic functions over level curves of certain other harmonic functions in the plane. Also, in broad outline, there are similarities with recent work by Armitage [4] in the half-space. However, there is little in common with respect to the methods employed, as that paper relies heavily on a passage technique (due to Huber [19] and Kuran [22]), which is special to the half-space.

This work was supported by a grant from the Department of Education for Northern Ireland. 
As in [14], we shall first give the general theory, and then conclude with specific applications (Sections 12-14). However, in view of the more difficult nature of the work, we shall attempt to illuminate the general exposition by concurrent reference to the two-dimensional strip.

\section{The framework}

Points of $\mathbf{R}^{n}$ will be denoted by capital letters such as $X, Y, Z, P$, or $Q$; in particular, $O$ will represent the origin of co-ordinates. When appropriate, $X$ will be written in terms of its co-ordinates

$$
X=\left(x_{1}, \ldots, x_{n}\right)=\left(X^{\prime}, x_{n}\right)
$$

where $X^{\prime} \in \mathbf{R}^{n-1}$. The closure and boundary of a subset $A$ of $\mathbf{R}^{n}$ will be denoted by $\bar{A}$ and $\partial A$ respectively, and, using $|X|$ to represent the Euclidean norm of $X$, we define

$$
B(X, r)=\left\{Y \in \mathbf{R}^{n}:|Y-X|<r\right\} .
$$

It will be convenient also to use $N(X)$ to denote the set of bounded open neighbourhoods of a point $X$ in $\mathbf{R}^{n}$.

We recall that a bounded domain $\omega \subset \mathbf{R}^{n}$ is called a Lipschitz domain if $\partial \omega$ can be covered by right circular cylinders whose bases have positive distances from $\partial \omega$, and corresponding to each cylinder $L$, there is a co-ordinate system $\left(\tilde{X}^{\prime}, \tilde{x}_{n}\right)$ with $\tilde{x}_{n}$-axis parallel to the axis of $L$, a function $f: \mathbf{R}^{n-1} \rightarrow \mathbf{R}$ and a real number $c$ such that

for all $\tilde{X}^{\prime}, \tilde{Y}^{\prime} \in \mathbf{R}^{n-1}$,

$$
\left|f\left(\tilde{X}^{\prime}\right)-f\left(\tilde{Y}^{\prime}\right)\right| \leqq c\left|\tilde{X}^{\prime}-\tilde{Y}^{\prime}\right|
$$

and

$$
L \cap \omega=\left\{X \in L: \tilde{x}_{n}>f\left(\tilde{X}^{\prime}\right)\right\}
$$

$$
L \cap \partial \omega=\left\{X \in L: \tilde{x}_{n}=f\left(\tilde{X}^{\prime}\right)\right\} .
$$

(The extra generality of non-tangentially accessible domains (see [21]) is unnecessary for the type of applications we have in mind.)

An account of the Perron-Wiener-Brelot generalized solution of the Dirichlet problem is given in Helms' book [18, Chapter 8], and we shall adopt his notation. Thus, if $f$ is resolutive on the boundary of an open set $W$, the Dirichlet solution is given by $H_{f}^{W}$.

Let $\Omega$ be an unbounded domain in $\mathbf{R}^{n}$ such that, for each $r>0$, there is an open set $W_{r}^{\prime} \supseteqq B(O, r)$ for which $\Omega_{r}^{\prime}=W_{r}^{\prime} \cap \Omega$ is a Lipschitz demain. To avoid having to deal repeatedly with it as a special case, we shall exclude the possibility of $\Omega=\mathbf{R}^{2}$. We now state a number of lemmas, whose proofs will be given in Sections 6 and 7 .

Lemma 1. There exist

(a) a Green kernel $G$ for $\Omega$ such that, if $X \in \Omega$, then $G(X,$.$) continuously vanishes$ on $\partial \Omega$, and 
(b) at least one positive harmonic function $h$ in $\Omega$ which continuously vanishes on $\partial \Omega$.

In view of (b) above, we define $h_{*}$ to be a (fixed) positive harmonic function in $\Omega$ which vanishes on $\partial \Omega$. We also let $v$ be a fixed (non-zero) Borel measure with compact support $E \subset \bar{\Omega}$.

Lemma 2. The function $G(X, Y) /\left\{h_{*}(X) h_{*}(Y)\right\}$ has a positive, symmetric, jointly continuous extension to $(\bar{\Omega} \times \bar{\Omega}) \backslash\{(X, Y): X=Y \in \partial \Omega\}$ (continuous in the extended sense at points of the diagonal of $\Omega \times \Omega)$, which we denote by $G^{*}(X, Y)$. Further, $h_{*}(.) G^{*}(., Y)$ is harmonic in $\Omega \backslash\{Y\}$.

We define

$$
\Phi(X)=\int_{E} G^{*}(X, Z) d v(Z) \quad(X \in \bar{\Omega} \backslash(E \cap \partial \Omega)),
$$

and extend $\Phi$ to be defined on $\bar{\Omega}$ by writing

$$
\Phi(X)=\liminf _{Y \rightarrow X} \Phi(Y) \quad(X \in E \cap \partial \Omega) .
$$

Clearly $\Phi$ is lower semicontinuous (1.s.c.) on $\bar{\Omega}$. We also have:

Lemma 3. The function $\Phi$ is positive on $\bar{\Omega}$, and $h_{*} \Phi$ is superharmonic in $\Omega$, harmonic in $\Omega \backslash E$ and continuously vanishes on $\partial \Omega \backslash E$.

Definition 1. Let $x$ denote the (positive, possibly infinite) infimum of $\Phi$ on $E$, and let $\varphi$ denote a (fixed) strictly decreasing mapping from $(0,+\infty)$ onto $(0, x)$ (which implies that $\varphi$ is continuous and invertible). Since $\Phi$ is 1.s.c. on $\bar{\Omega}$, there exists (for each $x>0$ ) an open set $W_{x}$ such that

$$
W_{x} \cap \bar{\Omega}=\{X \in \bar{\Omega}: \Phi(X)>\varphi(x)\} .
$$

We shall suppose that each $\Omega_{x}=W_{x} \cap \Omega$ is a Lipschitz domain, and that, if $x<w$, then $\bar{\Omega}_{w} \backslash W_{x}$ is the disjoint union of the closures of finitely many Lipschitz domains. This will certainly be the case in our applications. We abbreviate the sets $\partial \Omega_{x} \cap \Omega$ and $W_{x} \cap \partial \Omega$ to $\sigma_{x}$ and $\tau_{x}$ respectively, and denote harmonic measure with respect to $\Omega_{x}$ and $X \in \Omega_{x}$ by $\mu_{x, X}$. In view of [24, Théorème 25] and the fact that a cone internal to $\Omega_{w}$ with vertex at $Z \in \partial \Omega_{w}$ is non-thin at $Z$ (see, for example, [20, Lemma (3.6)]), it follows that $\partial \Omega_{x} \cap \partial \Omega \backslash \tau_{x}$ has $\mu_{x, X}$-measure zero for any $X \in \Omega_{x}$. The Green kernel for $\Omega_{x}$ will be denoted by $G_{x}$.

Lemma 4.
(a) $E \subseteq \bigcap_{x>0} \bar{\Omega}_{x}$;
(b) $\bar{\Omega}=\bigcup_{x>0} \bar{\Omega}_{x}$;
(c) $x<w \Rightarrow \bar{\Omega}_{x} \subset W_{w} \cap \bar{\Omega}$;
(d) $\Phi(X)=\varphi(x)$ for $X \in \sigma_{x}$. 
For suitable functions $f$ we define

and

$$
I_{f, x}(X)=\int_{\sigma_{x}} f(Z) d \mu_{x, X}(Z),
$$

$$
H_{f, x}(X)=H_{f}^{\Omega_{x}}(X)-\int_{\tau_{1}} f(Z) d \mu_{1, X}(Z),
$$

which are clearly harmonic in $\Omega_{\min \{x, 1\}}$, provided that the integrals are finite. It was shown in [14, Lemma 1] that the quotients $H_{f, x} / h_{*}$ and $I_{f, x} / h_{*}$ can be continuously defined on $W_{y} \cap \bar{\Omega}$, where $y=\min \{x, 1\}$. Denoting these extended functions respectively by $\mathscr{H}_{f, x}$ and $\mathscr{I}_{f, x}$, we define

$$
\mathscr{M}(f, x)=\int_{E} \mathscr{H}_{f, x}(X) d v(X)
$$

and

$$
\mathscr{N}(f, x)=\int_{E} \mathscr{I}_{f, x}(X) d v(X) .
$$

Let $s$ be subharmonic in $\Omega$ and extend it to $\bar{\Omega}$ by

$$
s(Z)=\limsup _{X \rightarrow Z} s(X) \quad(Z \in \partial \Omega) .
$$

If, for each $Z \in \partial \Omega$, there is a bounded neighbourhood of $Z$, whose intersection $\omega$ with $\Omega$ satisfies

(i) the restriction of $s$ to $\partial \omega$ is resolutive for $\omega$, and

(ii) $s \leqq H_{s}^{\omega}$ in $\omega$, then we say that $s \in \mathscr{L} \mathscr{D}$.

If $s \in \mathscr{L} \mathscr{D}$, then it follows from [15, Theorem 2 (i)] that $s$ is resolutive for every $\Omega_{x}$, and $s \leqq H_{s}^{\Omega_{x}}$ in $\Omega_{x}$. Hence $H_{s, x}, \mathscr{H}_{s, x}, \mathscr{M}(s, x), I_{s, x}, \mathscr{I}_{s, x}$, and $\mathscr{N}(s, x)$ all exist, and it is easy to see (cf. [14, Theorem 1]) that

(i) $\mathscr{M}(s, x)$ is an increasing $\left.{ }^{1}\right)$, real-valued function of $x$.

(ii) If also $s \leqq 0$ on $\partial \Omega$, then the same is true of $\mathscr{N}(s, x)$.

(iii) If $h$ is harmonic in $\Omega$ and continuous on $\bar{\Omega}$, then $\mathscr{M}(h, x)$ is a constant function of $x$.

Lemma 5. The function $F_{x}$, defined on $\bar{\Omega} \times \Omega_{x}$ by

$$
F_{x}(X, Y)=I_{h_{*}(\cdot) G^{*}(X, \cdot), x}(Y) / h_{*}(Y)
$$

has a jointly continuous extension to $\bar{\Omega} \times\left(W_{x} \cap \bar{\Omega}\right)$ such that $h_{*}(.) F_{x}(., Y)$ is harmonic in $\Omega_{x}$ for any $Y$. Further,

$$
G^{*}(X, Y)=F_{x}(X, Y) \quad\left(X \notin W_{x} \cap \bar{\Omega}\right),
$$

and

$$
G^{*}(X, Y)=F_{x}(X, Y)+\lim _{(P, Q) \rightarrow(X, Y)} G_{x}(P, Q) /\left\{h_{*}(P) h_{*}(Q)\right\}
$$

if $X, Y \in W_{x} \cap \bar{\Omega}$ and $X \neq Y$, the limit being unnecessary if both $X$ and $Y$ are in $\Omega_{x}$. The extended function of the above lemma will also be denoted by $F_{x}$.

\footnotetext{
1) We use increasing in the wide sense.
} 
We conclude this section by illustrating some of our definitions.

Example 1. Consider the two-dimensional case of the strip, so that

and

$$
\begin{gathered}
\Omega=(-1,1) \times \mathbf{R}, \quad E=[-1,1] \times\{0\} \\
d v\left(x_{1}, x_{2}\right)=8 \pi^{-2} \cos ^{2}\left(\frac{1}{2} \pi x_{1}\right) d x_{1} d \delta_{0}\left(x_{2}\right)
\end{gathered}
$$

$$
h_{*}\left(x_{1}, x_{2}\right)=2 \pi^{-1} \cos \left(\frac{1}{2} \pi x_{1}\right) \cosh \left(\frac{1}{2} \pi x_{2}\right),
$$

where $\delta_{0}$ is the Dirac measure at the origin of $\mathbf{R}$. The Green kernel for $\Omega$ is wellknown (see, for example, [7, Lemmas 3, 4] and use a simple conformal mapping); in particular,

$$
\begin{gathered}
G\left(\left(x_{1}, x_{2}\right),\left(y_{1}, 0\right)\right) \\
=2 \sum_{m=1}^{\infty} m^{-1} \sin \left[\frac{1}{2} m \pi\left(x_{1}+1\right)\right] \sin \left[\frac{1}{2} m \pi\left(y_{1}+1\right)\right] \exp \left(-\frac{1}{2} m \pi\left|x_{2}\right|\right) .
\end{gathered}
$$

If $x_{2} \neq 0$, then clearly the series converges uniformly in $y_{1}$, and so we can integrate term-by-term to obtain

$$
\begin{aligned}
\Phi\left(x_{1}, x_{2}\right) & =2 \int_{-1}^{1} G\left(\left(x_{1}, x_{2}\right),\left(y_{1}, 0\right)\right) \cos \left(\frac{1}{2} \pi y_{1}\right) /\left\{\cos \left(\frac{1}{2} \pi x_{1}\right) \cosh \left(\frac{1}{2} \pi x_{2}\right)\right\} d y_{1} \\
& =4 \operatorname{sech}\left(\frac{1}{2} \pi x_{2}\right) \exp \left(-\frac{1}{2} \pi\left|x_{2}\right|\right) \\
& =8\left\{1+\exp \left(\pi\left|x_{2}\right|\right)\right\}^{-1} .
\end{aligned}
$$

This remains valid for $x_{2}=0$ by the 1 .s. continuity of $\Phi$. Thus $x=4$ and, defining $\varphi:(0,+\infty) \rightarrow(0,4)$ by

$$
\varphi(x)=8\{1+\exp (\pi x)\}^{-1},
$$

it follows that $\Omega_{x}=(-1,1) \times(-x, x)$. The assumptions of Definition 1 are now easily seen to hold.

\section{The generalized mean}

If $s$ is subharmonic in $\Omega$, then the measure associated with $s$ in $\Omega$ is given by $\mu_{s}=\gamma_{n} \Delta s$, where

$$
\gamma_{2}=(2 \pi)^{-1}, \quad \gamma_{n}=\left\{(n-2) c_{n}\right\}^{-1} \quad(n \geqq 3),
$$

$c_{n}$ denoting the surface area of $\partial B(O, 1)$, and $\Delta s$ is the distributional Laplacian of $s$ in $\Omega$. The following result associates a second measure, defined on $\partial \Omega$, with $s$.

Theorem 1. If $s \in \mathscr{L} \mathscr{D}$, then there exists a unique measure $\lambda_{s}$ on $\partial \Omega$ such that the least harmonic majorant of $s$ in $\Omega_{x}$ is given by

$$
H_{s}^{\Omega_{x}}(Y)-h_{*}(Y) \int_{\tau_{x}}\left\{G^{*}(X, Y)-F_{x}(X, Y)\right\} d \lambda_{s}(X) .
$$


(We remark that, if $s$ is subharmonic in an open set containing $\bar{\Omega}$, then the least harmonic majorant of $s$ in $\Omega_{x}$ is given by $H_{s}^{\Omega_{x}}$, and so $\lambda_{s}$ is the zero measure on $\partial \Omega$.)

Definition 2. We introduce a modified mean $\mathscr{M}^{*}(s, x)$ for $s \in \mathscr{L} \mathscr{D}$, given by

$$
\mathscr{M}^{*}(s, x)=\mathscr{M}(s, x)+\int_{1}^{x} \lambda_{s}\left(\tau_{t}\right) d \varphi(t),
$$

where the latter term is a Riemann-Stieltjes integral.

Example 2. Following on from Example 1, we deduce from [14, Section 9] that

$$
\begin{gathered}
\mathscr{M}(s, x)=2 \pi^{-1} \operatorname{sech}\left(\frac{1}{2} \pi x\right) \int_{-1}^{1} \cos \left(\frac{1}{2} \pi x_{1}\right)\left\{s\left(x_{1}, x\right)+s\left(x_{1},-x\right)\right\} d x_{1} \\
+\int_{1}^{x} \operatorname{sech}^{2}\left(\frac{1}{2} \pi t\right) \int_{-t}^{t} \cosh \left(\frac{1}{2} \pi x_{2}\right)\left\{s\left(-1, x_{2}\right)+s\left(1, x_{2}\right)\right\} d x_{2} d t .
\end{gathered}
$$

Since the derivative of $\varphi(x)$ is $-2 \pi \operatorname{sech}^{2}\left(\frac{1}{2} \pi x\right)$, we have

$$
\begin{gathered}
\mathscr{M}^{*}(s, x)=2 \pi^{-1} \operatorname{sech}\left(\frac{1}{2} \pi x\right) \int_{-1}^{1} \cos \left(\frac{1}{2} \pi x_{1}\right)\left\{s\left(x_{1}, x\right)+s\left(x_{1},-x\right)\right\} d x_{1} \\
+\int_{1}^{x} \operatorname{sech}^{2}\left(\frac{1}{2} \pi t\right)\left[\int_{-t}^{t} \cosh \left(\frac{1}{2} \pi x_{2}\right)\left\{s\left(-1, x_{2}\right)+s\left(1, x_{2}\right)\right\} d x_{2}\right. \\
\left.-2 \pi \lambda_{s}(\{-1,1\} \times(-t, t))\right] d t .
\end{gathered}
$$

The following is a generalization of Nevanlinna's first fundamental theorem for subharmonic functions in $\mathbf{R}^{n}$ (see [16, p. 127]).

Theorem 2. If $s \in \mathscr{L} \mathscr{D}$, then

$$
\mathscr{M}^{*}(s, x)=\mathscr{N}(s, 1)-\int_{1}^{x} \int_{\Omega_{t}} h_{*}(Z) d \mu_{s}(Z) d \varphi(t) .
$$

Proofs of Theorems 1 and 2 may be found in Sections 8 and 9, respectively.

\section{General results}

Theorem 2 is used to deduce the main results of this paper.

Theorem 3. Let $s \in \mathscr{L} \mathscr{D}$. Then

(i) $\mathscr{M}^{*}(s, x)$ is increasing as a function of $x$ and convex as a function of $\varphi(x)$ on $(0,+\infty)$;

(ii) if $w>y>0$ and $s$ is harmonic in $\Omega_{w} \backslash \bar{\Omega}_{y}$, then $\mathscr{M}^{*}(s, x)$ is a linear function of $\varphi(x)$ on $[y, w]$;

(iii) $\mathscr{M}^{*}(s, x)$ is constant on $(0,+\infty)$ if and only if $s$ is harmonic in $\Omega$. 
Theorem 4. If $s \in \mathscr{L} \mathscr{D}$, then the following are equivalent:

(i) $s$ has a harmonic majorant in $\Omega$;

(ii) $\mathscr{M}^{*}(s, x)$ is bounded above on $(0,+\infty)$;

(iii) $\int_{\Omega \backslash \Omega_{1}} h_{*}(X) \Phi(X) d \mu_{s}(X)<+\infty$.

Theorems 3 and 4 show that $\mathscr{M}^{*}(s, x)$ has "ideal" properties; that is, it behaves exactly like the ordinary spherical mean of subharmonic functions in $\mathbf{R}^{n}$ (of which it is a generalization). The major disadvantage of this mean is that $\lambda_{s}$ has to be defined in a rather indirect fashion. Thus there is a case for discussing also the (slightly less satisfactory) properties of $\mathscr{M}(s, x)$, some of which have already been given in [14].

Theorem 5. (i) If $s \in \mathscr{L} \mathscr{D}$ and $\mathscr{M}(s, \mathrm{x})$ is bounded above on $(0,+\infty)$, then $s$ has a harmonic majorant in $\Omega$.

(ii) Let $s$ be subharmonic in an open set $W$ containing $\bar{\Omega}$. Then $s$ has a harmonic majorant in $\Omega$ if and only if $\mathscr{M}(s, x)$ is bounded above on $(0,+\infty)$.

Part (i) holds since $\mathscr{M}^{*}(s, x) \leqq \mathscr{M}(s, x)$ (see Definition $2 ; \varphi$ is decreasing), and generalizes [14, Theorem 2]. Part (ii) is identical to [14, Theorem 3] and is immediate since, in this case $\lambda_{s} \equiv 0$.

Convexity results for $\mathscr{M}(s, x)$ were not considered in [14], but are now also easily derived.

Theorem 6. (i) If $s \in \mathscr{L} \mathscr{D}$, then $\mathscr{M}(s, x)$ is increasing as a function of $x$, and convex as a function of $\varphi(x)$ on $(0,+\infty)$.

(ii) If also $s \leqq 0$ on $\partial \Omega$, then $\mathscr{N}(s, x)$ is increasing as a function of $x$, and convex as a function of $\varphi(x)$ on $(0,+\infty)$.

Theorems 3, 4 and 6 are proved in Section 10.

\section{Variant means}

Analogous results for variants of the mean $\mathscr{N}(s, x)$ are now given.

Theorem 7. If $s$ is a non-negative subharmonic function in $\Omega$ which continuously vanishes on $\partial \Omega$, and $1 \leqq p<+\infty$, then the mean

$$
\mathscr{N}_{p}(s, x)=\left\{\mathscr{N}\left(h_{*}^{1-p} s^{p}, x\right)\right\}^{1 / p}
$$

is real-valued, convex as a function of $\varphi(x)$, and increasing as a function of $x>0$.

Theorem 8. If $u$ is a positive superharmonic function in $\Omega$, and $p \in(-\infty, 0) \cup$ $(0,1)$, then $\mathcal{N}_{p}(u, x)$ is real-valued, concave as a function of $\varphi(x)$, and decreasing as a function of $x>0$. 
Theorem 9. If $s \in \mathscr{L} \mathscr{D}$ and $s \leqq 0$ on $\partial \Omega$, then the "mean"

$$
\mathscr{N}_{\infty}(s, x)=\sup \left\{s(X) / h_{*}(X): X \in \sigma_{x}\right\}
$$

is real-valued, convex as a function of $\varphi(x)$, and increasing as a function of $x>0$.

Theorem 10. If $s \in \mathscr{L} \mathscr{D}$ and $s \leqq 0$ on $\partial \Omega$, then the mean

$$
\mathscr{N}_{E}(s, x)=\log \mathscr{N}\left(h_{*} \exp \left(s / h_{*}\right), x\right)
$$

is real-valued, convex as a function of $\varphi(x)$, and increasing as a function of $x>0$.

The proofs of these theorems are closely related, and are based on a technique of Fugard [12, Chapter 2] (or see [5, Theorems 7 and 8]). We shall illustrate this by giving the proof of Theorem 10 in Section 11. Theorem 9 is a generalization of Hadamard's Three Circles Theorem, and can equivalently be stated in terms of the infimum of $u / h_{*}$ over $\sigma_{x}$ for suitable superharmonic functions $u$. It is a little easier to prove, and the maximum principle can be used to establish the monotonicity part of the result.

\section{Proofs of Lemmas $1-4$}

6.1. We shall make use of the following results.

Theorem A. (Boundary Harnack principle.) Let $\Omega^{\prime}$ be a bounded Lipschitz domain of which $P$ is a fixed point, $A$ be a relatively open subset of $\partial \Omega^{\prime}$, and $W^{\prime}$ be a subdomain of $\Omega^{\prime}$ satisfying $\partial \Omega^{\prime} \cap \partial W^{\prime} \subseteq A$. Then there is a constant $c$ such that, if $h_{1}$ and $h_{2}$ are two positive harmonic functions in $\Omega^{\prime}$ vanishing on $A$ and $h_{1}(P)=h_{2}(P)$, then $h_{1}(X) \leqq c h_{2}(X)$ for all $X \in W^{\prime}$.

Theorem B. If $h_{1}$ and $h_{2}$ are positive harmonic functions on a bounded Lipschitz domain $\Omega^{\prime}$ vanishing on a relatively open subset $A$ of $\partial \Omega^{\prime}$, then $h_{1} / h_{2}$ can be continuously extended to a strictly positive function defined on $\Omega^{\prime} \cup A$.

For Theorem A we refer to either Dahlberg [10, Theorem 4] or Wu [30, Theorem 1]. If the set $A$ is empty, then the result reduces to the usual Harnack inequality [18, Theorem 2.16]. Alternative proofs for Theorem B can be found in $[21,(7.9)]$ and $[6$, Theorem 2].

6.2. To prove Lemma 1 , first note that $\Omega$ has a Green kernel. If $n \geqq 3$, this is immediate; if $n=2$, choose $r$ such that $W_{r}^{\prime} \cap \partial \Omega$ is non-empty. Since $\Omega_{r}^{\prime}$ is Lipschitz, there exist $Y$ and $\varepsilon>0$ such that $\bar{B}(Y, \varepsilon) \subseteq W_{r}^{\prime} \backslash \bar{\Omega}$, whence $\Omega \subseteq \mathbf{R}^{2} \backslash \bar{B}(Y, \varepsilon)$ and so $\Omega$ has a Green kernel.

Denoting this kernel by $G$ and letting $X \in \Omega$, we show that $G(X,$.$) vanishes$ on $\partial \Omega$. Fix $r$ such that $X \in \Omega_{r}^{\prime}$, let $G_{r}^{\prime}$ be the Green kernel for $\Omega_{r}^{\prime}$, and define $f_{X}$ on 
$\partial \Omega_{r}^{\prime}$ by setting it equal to $G(X,$.$) on \partial \Omega_{r}^{\prime} \cap \Omega$ and 0 elsewhere. Then the function

$$
s(Y)= \begin{cases}G(X, Y)-G_{r}^{\prime}(X, Y)-H_{f_{X}}^{\Omega_{r}^{\prime}}(Y) & \left(Y \in \Omega_{r}^{\prime}\right) \\ 0 & \left(Y \in \Omega \backslash \Omega_{r}^{\prime}\right)\end{cases}
$$

is easily seen to be a non-negative subharmonic minorant of $G(X, Y)$ in $\Omega$ (since $\Omega_{r}^{\prime}$ is regular) and so is identically zero. Since $G_{r}^{\prime}(X,$.$) and H_{f_{x}}^{\Omega_{r}^{\prime}}$ vanish on $W_{r}^{\prime} \cap \partial \Omega$, so does $G(X,$.$) , and since r$ may be arbitrarily large, part (a) is proved.

To prove (b), let $Q$ be a fixed point in $\Omega$ and $\left(Y_{m}\right)$ be an unbounded sequence of points in $\Omega$. By choosing a suitable subsequence if necessary (compactness argument), we may assume that $\left(Y_{m}\right)$ converges to a Martin boundary point of $\Omega$. If $\partial \Omega$ is empty, the lemma is trivially true. Otherwise, let $r$ and $R$ be such that $\bar{\Omega}_{r}^{\prime} \subset W_{R}^{\prime}$ and $Q \in \Omega_{R}^{\prime}$, and such that $W_{r}^{\prime} \cap \partial \Omega$ is non-empty. Fix $P \in \Omega \backslash \bar{\Omega}_{R}^{\prime}$. From Theorem $\mathrm{A}$ there is a constant $c$ such that

whence

$$
G\left(Y_{m}, X\right) / G\left(Y_{m}, Q\right) \leqq c G(P, X) / G(P, Q) \quad\left(X \in \Omega_{r}^{\prime}\right)
$$

$$
h(X)=\lim _{m \rightarrow \infty} G\left(Y_{m}, X\right) / G\left(Y_{m}, Q\right) \leqq c^{\prime} G(P, X) \quad\left(X \in \Omega_{r}^{\prime}\right) .
$$

Thus $h$ is a positive harmonic function in $\Omega_{r}^{\prime}$ which vanishes on $W_{r}^{\prime} \cap \partial \Omega$. Since $r$ may be arbitrarily large, (b) is proved.

6.3. We now prove Lemma 2 . Let $X_{0}, Y_{0} \in \bar{\Omega}$. Joint continuity clearly holds at $\left(X_{0}, Y_{0}\right)$ unless at least one of $X_{0}, Y_{0}$ is in $\partial \Omega$. We shall consider the case where both $X_{0}$ and $Y_{0}$ are in $\partial \Omega$ and $X_{0} \neq Y_{0}$, the case where only one of $X_{0}, Y_{0}$ is in $\partial \Omega$ being similar and easier. It is clearly sufficient to show that $G^{*}(X, Y)$ has a limit as $(X, Y)$ tends to $\left(X_{0}, Y_{0}\right)$ from within $\Omega \times \Omega$.

Let $U_{i} \in N\left(X_{0}\right)$ and $V_{i} \in N\left(Y_{0}\right)(i=1,2,3)$ be such that

(i) $\bar{U}_{3} \subset U_{2} \subset \bar{U}_{2} \subset U_{1}$ and similarly for $V_{i}$;

(ii) $\bar{U}_{1} \cap \bar{V}_{1}=\emptyset$;

(iii) the sets $U_{i}^{\prime}=U_{i} \cap \Omega$ and $V_{i}^{\prime}=V_{i} \cap \Omega$ are Lipschitz domains (this is possible because $X_{0}, Y_{0} \in W_{r}^{\prime}$ for sufficiently large $r$ ). We denote harmonic measure for $U_{i}^{\prime}$ and $X \in U_{i}^{\prime}$ by $\lambda_{i, X}$, and for $V_{i}^{\prime}$ and $Y \in V_{i}^{\prime}$ by $v_{i, Y}$.

In view of (ii), $G(X, Y)$ is bounded above, by $c$ say, for $(X, Y)$ in $U_{1}^{\prime} \times V_{1}^{\prime}$. From Theorem A

$$
\begin{aligned}
G(X, Y) & =\int_{\partial U_{1} \cap \Omega} G(Z, Y) d \lambda_{1, X}(Z) \\
& \leqq c \lambda_{1, X}\left(\partial U_{1} \cap \Omega\right) \leqq c^{i} h_{*}(X)
\end{aligned}
$$

for $X \in U_{2}^{\prime}$ and $Y \in V_{1}^{\prime}$. Repeating this argument, we obtain

$$
G(X, Y) / h_{*}(X)=\int_{\partial V_{1} \cap \Omega} G(X, Z) / h_{*}(X) d v_{1, Y}(Z) \leqq c^{i i} h_{*}(Y)
$$

for $X \in U_{2}^{\prime}$ and $Y \in V_{2}^{\prime}$. 
Let $\varepsilon^{\prime}>0$. It follows from (3) and the joint continuity of $G$ in $\Omega \times \Omega$ that there exists $\delta>0$ such that

$$
\left|G\left(X_{1}, Y\right)-G\left(X_{2}, Y\right)\right|<\varepsilon^{\prime} \quad\left(Y \in \partial V_{2} \cap \Omega\right)
$$

for $X_{1}, X_{2} \in \partial U_{2} \cap \Omega$ satisfying $\left|X_{1}-X_{2}\right|<\delta$. Again using Theorem $\mathrm{A}$

$$
\left|G\left(X_{1}, Y\right)-G\left(X_{2}, Y\right)\right| \leqq \varepsilon^{\prime} v_{2, Y}\left(\partial V_{2} \cap \Omega\right)<c^{i i i} \varepsilon^{\prime} h_{*}(Y) \quad\left(Y \in V_{3}^{\prime}\right) .
$$

From Theorem B, the functions

$$
\left\{G(X, .) / h_{*}(.): X \in \partial U_{2} \cap \Omega\right\}
$$

have continuous extensions to $V_{3}^{\prime}$. Hence, from (3), (4) and the fact that $\partial U_{2} \cap \Omega$ is relatively compact, we can apply the Arzelà-Ascoli theorem to see that they are equicontinuous on $\bar{V}_{3}^{\prime}$.

Let $\varepsilon>0$. Then there exists $V_{\varepsilon} \in N\left(Y_{0}\right)$ such that $\bar{V}_{\varepsilon} \subset V_{3}$ and

$$
\left|G\left(X, Y_{1}\right) / h_{*}\left(Y_{1}\right)-G\left(X, Y_{2}\right) / h_{*}\left(Y_{2}\right)\right|<\varepsilon \quad\left(X \in \partial U_{2} \cap \Omega\right)
$$

for $Y_{1}, Y_{2} \in V_{\varepsilon}^{\prime}=V_{\varepsilon} \cap \Omega$, and so

$$
\left|G\left(X, Y_{1}\right) / h_{*}\left(Y_{1}\right)-G\left(X, Y_{2}\right) / h_{*}\left(Y_{2}\right)\right| \leqq \varepsilon \lambda_{2, X}\left(\partial U_{2} \cap \Omega\right)<c^{i v} \varepsilon h_{*}(X)
$$

for $X \in U_{3}^{\prime}$. Thus we have

$$
\left|G^{*}\left(X, Y_{1}\right)-G^{*}\left(X, Y_{2}\right)\right|<c^{i v} \varepsilon \quad\left(X \in U_{3}^{\prime} ; Y_{1}, Y_{2} \in V_{\varepsilon}^{\prime}\right) .
$$

Correspondingly, we obtain $U_{\varepsilon}^{\prime}$ such that

and so

$$
\left|G^{*}\left(X_{1}, Y\right)-G^{*}\left(X_{2}, Y\right)\right|<c^{v} \varepsilon \quad\left(X_{1}, X_{2} \in U_{\varepsilon}^{\prime} ; Y \in V_{3}^{\prime}\right),
$$

$$
\left|G^{*}\left(X_{1}, Y_{1}\right)-G^{*}\left(X_{2}, Y_{2}\right)\right|<\left(c^{i v}+c^{v}\right) \varepsilon \quad\left(X_{1}, X_{2} \in U_{\varepsilon}^{\prime} ; Y_{1}, Y_{2} \in V_{\varepsilon}^{\prime}\right),
$$

where $c^{i v}$ and $c^{v}$ are independent of $\varepsilon$. A completeness argument now shows that $G^{*}(X, Y)$ has a limit as $(X, Y) \rightarrow\left(X_{0}, Y_{0}\right)$.

The harmonicity of $h_{*}(.) G^{*}(., Y)$ in $\Omega \backslash\{Y\}$ is clear if $Y \in \Omega$. If $Y \in \partial \Omega$, let $\left(Y_{m}\right)$ be a sequence of points in $\Omega$, converging to $Y$. In view of the joint continuity of $G^{*}$, the functions $h_{*}(.) G^{*}\left(., Y_{m}\right)$ are locally uniformly bounded in $\Omega$ and so their limit is harmonic in $\Omega$ (see [18; Theorem 2.18]).

The positivity of $G^{*}$ is a consequence of Theorem $\mathrm{B}$, and the symmetry is obvious from the symmetry of $G$.

6.4. In Lemma 3, since $G^{*}$ is positive and $v$ is non-zero, the positivity of $\Phi$ need be checked only at points $Z$ of $E \cap \partial \Omega$. To do this, we choose $r$ such that $E \subset W_{r}^{\prime}$ and apply [24, Théorème $\left.7^{\prime}-16\right]$ and Theorem $B$ to see that

$$
\Phi(Z)=\left\{\liminf _{X \rightarrow Z} h_{*}(X) \Phi(X) / G_{r}^{\prime}(X, Q)\right\}\left\{\lim _{X \rightarrow Z} G_{r}^{\prime}(X, Q) / h_{*}(X)\right\}>0,
$$

where $Q$ is an arbitrary point of $\Omega_{r}^{\prime}$. 
Let $v_{1}$ and $v_{2}$ be the restrictions of $v$ to $\partial \Omega \cap E$ and $\Omega \cap E$ respectively, and define

$$
d v_{3}(Y)=\left\{h_{*}(Y)\right\}^{-1} d v_{2}(Y) .
$$

In view of Lemma $2, h_{*} \Phi$ is clearly finite on $\Omega \backslash E$, and so the function $G v_{3}$ is a potential in $\Omega$. It is immediate also from Lemma 2 that $h_{*} \Phi$ vanishes on $\partial \Omega \backslash E$, and so it remains only to show that

$$
\int_{\partial \Omega \cap E} h_{*}(.) G^{*}(., Y) d v_{1}(Y)
$$

is harmonic in $\Omega$. In fact, Lemma 2 ensures that this function is continuous in $\Omega$ and Fubini's theorem shows that the mean-value equality holds for all sufficiently small spheres centred at any $X \in \Omega$.

6.5. Lemma 4 is straightforward to establish. Since, for each $x, \varphi(x)<x$, it follows that

$$
E \leqq W_{x} \cap \bar{\Omega} \subseteq \bar{\Omega}_{x},
$$

and so (a) holds. Part (b) is true because $\Phi$ is positive in $\bar{\Omega}$. Since $\Phi$ is continuous in $\bar{\Omega} \backslash E$, we have $\Phi(X) \geqq \varphi(x)$ for $X \in \bar{\Omega}_{x}$, and so

$$
x<w \Rightarrow \varphi(x)>\varphi(w) \Rightarrow \bar{\Omega}_{x} \subset W_{w} \cap \bar{\Omega},
$$

proving (c). Part (d) follows from the continuity of $\Phi$ at points of $\sigma_{x}$.

\section{Proof of Lemma 5}

Once the joint continuity of $F_{x}$ is established, the harmonicity of $h_{*}(.) F_{x}(., Y)$ follows easily as in Lemma 2 . Let

and

$$
S_{1}=\left(\Omega \backslash \bar{\Omega}_{x}\right) \times \Omega_{x}, \quad S_{2}=\Omega_{x} \times \Omega_{x}
$$

$$
\left(X_{0}, Y_{0}\right) \in \bar{\Omega} \times\left(W_{x} \cap \bar{\Omega}\right) .
$$

We shall show that, as $(X, Y)$ tends to $\left(X_{0}, Y_{0}\right)$ from within $S_{1} \cup S_{2}$, the function $F_{x}(X, Y)$ tends to a limit, which equals $F_{x}\left(X_{0}, Y_{0}\right)$ if $Y_{0} \in \Omega_{x}$. Our proof falls naturally into three parts.

Case I: $X_{0} \in \bar{\Omega} \backslash \bar{\Omega}_{x}$. For $X \in \Omega \backslash \bar{\Omega}_{x}$, the function $h_{*}(.) G^{*}(X,$.$) is harmonic$ in $\Omega_{x}$, continuous in $\bar{\Omega}_{x}$ and valued zero on $\tau_{x}$ (see Lemma 2). Thus, if $(X, Y) \in S_{1}$, then

$$
F_{x}(X, Y)=h_{*}(Y) G^{*}(X, Y) / h_{*}(Y)=G^{*}(X, Y) .
$$

As $(X, Y) \rightarrow\left(X_{0}, Y_{0}\right)$, we have $F_{x}(X, Y) \rightarrow G^{*}\left(X_{0}, Y_{0}\right)$, and if $Y_{0} \in \Omega_{x}$, then $G^{*}\left(X_{0}, Y_{0}\right)=F_{x}\left(X_{0}, Y_{0}\right)$ as required. 
Case II: $X_{0} \in \Omega_{x} \cup \tau_{x}=W_{x} \cap \bar{\Omega}$. Let $\varepsilon>0$. From the joint continuity of $G^{*}$ there exists $U_{\varepsilon} \in N\left(X_{0}\right)$ such that $\bar{U}_{\varepsilon} \subset W_{x}$ and

$$
\left|G^{*}\left(X_{1}, Z\right)-G^{*}\left(X_{2}, Z\right)\right|<\varepsilon
$$

for $X_{1}$ and $X_{2}$ in $U_{\varepsilon} \cap \bar{\Omega}$ and $Z$ in $\sigma_{x}$. Hence

$$
\left|F_{x}\left(X_{1}, Y\right)-F_{x}\left(X_{2}, Y\right)\right|<\varepsilon
$$

for $X_{1}$ and $X_{2}$ in $U_{\varepsilon} \cap \bar{\Omega}$ and $Y \in \Omega_{x}$. Since

$$
G(X, Y)=I_{G(X, \cdot), x}(Y)+G_{x}(X, Y) \quad\left(X, Y \in \Omega_{x}\right),
$$

it is clear that $F_{x}$ is symmetric in $\Omega_{x} \times \Omega_{x}$ and so there also exists $V_{\varepsilon} \in N\left(Y_{0}\right)$ such that $\bar{V}_{\varepsilon} \subset W_{x}$ and

$$
\left|F_{x}\left(X, Y_{1}\right)-F_{x}\left(X, Y_{2}\right)\right|<\varepsilon
$$

for $X$ in $\Omega_{x}$ and $Y_{1}$ and $Y_{2}$ in $V_{\varepsilon} \cap \Omega$. If we let $X_{3} \in U_{\varepsilon} \cap \Omega$, then

$$
\begin{aligned}
\left|F_{x}\left(X_{1}, Y_{1}\right)-F_{x}\left(X_{2}, Y_{2}\right)\right| & \leqq\left|F_{x}\left(X_{1}, Y_{1}\right)-F_{x}\left(X_{3}, Y_{1}\right)\right| \\
+\mid F_{x}\left(X_{3}, Y_{1}\right) & -F_{x}\left(X_{3}, Y_{2}\right)|+| F_{x}\left(X_{3}, Y_{2}\right)-F_{x}\left(X_{2}, Y_{2}\right) \mid \\
& <3 \varepsilon
\end{aligned}
$$

for $X_{1}$ and $X_{2}$ in $U_{\varepsilon} \cap \bar{\Omega}$ and $Y_{1}, Y_{2} \in V_{\varepsilon} \cap \Omega$. A completeness argument shows that $F_{x}$ has a limit as $(X, Y) \rightarrow\left(X_{0}, Y_{0}\right)$. If $Y_{0} \in \Omega_{x}$, then choose $\left(X_{2}, Y_{2}\right)=\left(X_{0}, Y_{0}\right)$ to see that the limit is, in fact, $F_{x}\left(X_{0}, Y_{0}\right)$. Also, (1) now follows from (5) and Lemma 2.

Case III: $X_{0} \in \bar{\Omega}_{x} \backslash W_{x}$. This is the most difficult case to prove. Let $Q, Y \in \Omega_{x}$. If $w>x$, then, as has already been observed in Section $2, \Omega_{w} \backslash \bar{\Omega}_{x}$ is non-thin at $X_{0}$ in the minimal fine topology for $\Omega_{w}$, whence

$$
\int_{\sigma_{x} X \rightarrow X_{0}} \lim _{w}(X, Z) / G_{w}(X, Q) d \mu_{x, Y}(Z)=\lim _{X \rightarrow X_{0}} G_{w}(X, Y) / G_{w}(X, Q),
$$

or, in view of Theorem B,

$$
\int_{\sigma_{x}} \lim _{X \rightarrow X_{0}} G_{w}(X, Z) / h_{*}(X) d \mu_{x, Y}(Z)=\lim _{X \rightarrow X_{0}} G_{w}(X, Y) / h_{*}(X) .
$$

Also, from II, $F_{w}$ is jointly continuous in $\left(W_{w} \cap \bar{\Omega}\right) \times\left(W_{w} \cap \bar{\Omega}\right)$ and $h_{*}(.) F_{w}\left(X_{0},.\right)$ is harmonic in $\Omega_{w}$, and so

$$
\int_{\sigma_{x}} h_{*}(Z) F_{w}\left(X_{0}, Z\right) d \mu_{x, Y}(Z)=h_{*}(Y) F_{w}\left(X_{0}, Y\right) .
$$

Thus, from (1), (6) and (7),

$$
F_{x}\left(X_{0}, Y\right)=\int_{\sigma_{x}} h_{*}(Z) G^{*}\left(X_{0}, Z\right) d \mu_{x, Y}(Z) / h_{*}(Y)=G^{*}\left(X_{0}, Y\right) \quad\left(Y \in \Omega_{x}\right) .
$$

The lemma will follow if we show that

$$
F_{x}(X, Y) \rightarrow G^{*}\left(X_{0}, Y_{0}\right) \quad\left((X, Y) \rightarrow\left(X_{0}, Y_{0}\right) ;(X, Y) \in S_{1} \cup S_{2}\right) .
$$


This is clearly true as $(X, Y)$ tends to $\left(X_{0}, Y_{0}\right)$ from within $S_{1}$, since $F_{x}=G^{*}$ there. If $(X, Y) \in S_{2}$, then it follows from (1) that we need only prove

$$
G_{x}(X, Y) /\left\{h_{*}(X) h_{*}(Y)\right\} \rightarrow 0 \quad\left((X, Y) \rightarrow\left(X_{0}, Y_{0}\right)\right) .
$$

We use the non-thinness of $\Omega_{w} \backslash \bar{\Omega}_{x}$ at $X_{0}$ and [24, Théorème 11] to observe that

$$
G_{x}(X, Q) / G_{w}(X, Q) \rightarrow 0 \quad\left(X \rightarrow X_{0} ; X \in \Omega_{x}\right)
$$

and so, since $G_{w}(X, Q) / h_{*}(X)$ has a positive finite limit at $X_{0}$ (see Theorem B when $X_{0} \in \partial \Omega$ ),

$$
G_{x}(X, Q) / h_{*}(X) \rightarrow 0 \quad\left(X \rightarrow X_{0} ; X \in \Omega_{x}\right) .
$$

Now, since $Y_{0} \in W_{x} \cap \bar{\Omega}$ and $Q \in \Omega_{x}$, we can choose $z<y<x$ such that $Y_{0}$ is in $W_{z} \cap \bar{\Omega}$ and $Q \in \Omega_{y}$. Thus we may apply Theorem A with $\Omega=\Omega_{y}$ and $\Omega_{0}=\Omega_{z}$ to obtain the existence of a positive constant $c$ such that

and so

$$
G_{x}(X, Y) / G_{x}(X, Q) \leqq c h_{*}(Y) / h_{*}(Q)
$$

$$
G_{x}(X, Y) /\left\{h_{*}(X) h_{*}(Y)\right\} \leqq c^{\prime} G_{x}(X, Q) / h_{*}(X)
$$

for $X \in \Omega_{x} \backslash \Omega_{y}$ and $Y \in \Omega_{z}$. Combining (9) and (10) yields (8) as required.

\section{Proof of Theorem 1}

8.1. We recall (see [20, Theorem (4.2)]) that, if $\Omega^{\prime}$ is a Lipschitz domain, then every Martin boundary point of $\Omega^{\prime}$ is minimal, and the set $\Delta_{1}$ of Martin boundary points of $\Omega^{\prime}$ can be put into one-to-one correspondence with $\partial \Omega^{\prime}$ in such a way that the Martin topology on $\Omega^{\prime} \cup \Delta_{1}$ is equivalent to the Euclidean topology on $\bar{\Omega}^{\prime}$.

Lemma 6. Let $h$ be a non-negative harmonic function in a Lipschitz domain $\Omega^{\prime}$, and let $\mu$ be the measure on $\partial \Omega^{\prime}$ associated with it in the Martin representation. If $A$ is a relatively open subset of $\partial \Omega^{\prime}$, then $h$ vanishes continuously on $A$ if and only if $\mu(A)=0$.

The "only if" part follows from [30, Lemma 10]. The "if" part is trivial if $A$ is empty. Otherwise, let $Z \in A$ and choose $W \in N(Z)$ such that $\Omega^{\prime \prime}=W \cap \Omega^{\prime}$ is a domain and $\partial \Omega^{\prime \prime} \cap \partial \Omega^{\prime} \subset A$. Let $Q \in \Omega^{\prime} \backslash \bar{\Omega}^{\prime \prime}$ and $P \in \Omega^{\prime \prime}$. For a small positive value of $\varepsilon$, we can now apply Theorem A with $\Omega^{\prime}$ replaced by $\Omega^{\prime} \backslash \bar{B}(Q, \varepsilon)$ to deduce that

$$
K(Y, X) \leqq c K(Y, P) G^{\prime}(Q, X) / G^{\prime}(Q, P)
$$

for $Y \in \partial \Omega^{\prime} \backslash A$ and $X \in \Omega^{\prime \prime}$, where $G^{\prime}$ is the Green kernel for $\Omega^{\prime}$ and $K(.,$.$) denotes$ the Martin kernel on $\partial \Omega^{\prime} \times \Omega^{\prime}$ (by the Poincaré-Zaremba cone criterion [18, Theorem 8.27], $G(Q,$.$) vanishes on \partial \Omega^{\prime}$, and it is shown in [20] that $K(Y,$.$) van-$ 
ishes on $\left.\partial \Omega^{\prime} \backslash\{Y\}\right)$. Integrating both sides of (11) with respect to $d \mu(Y)$, it follows that

$$
h(X) \leqq c^{\prime} h(P) G^{\prime}(Q, X) \quad\left(X \in \Omega^{\prime \prime}\right)
$$

and so $h$ vanishes at $Z$ as required.

Lemma 7. If $\lambda$ and $\mu$ are measures on $\tau_{x}$ such that, for all $Y \in \Omega_{x}$, we have

$$
\int_{\tau_{x}}\left\{G^{*}(X, Y)-F_{x}(X, Y)\right\} d \lambda(X)=\int_{\tau_{x}}\left\{G^{*}(X, Y)-F_{x}(X, Y)\right\} d \mu(X),
$$

then $\lambda=\mu$.

Let

and define $\lambda^{\prime}$ on $\tau_{x}$ by

$$
h_{\lambda}(Y)=h_{*}(Y) \int_{\tau_{x}}\left\{G^{*}(X, Y)-F_{x}(X, Y)\right\} d \lambda(X),
$$

$$
d \lambda^{\prime}(X)=\left\{\lim _{Z \rightarrow X} G_{x}(Z, Q) / h_{*}(Z)\right\} d \lambda(X)
$$

where $Q$ is a fixed point of $\Omega_{x}$ (see Theorem B). From Lemmas 2 and $5, h_{\lambda}$ is harmonic in $\Omega_{x}$ and, from (1),

$$
\begin{aligned}
h_{\lambda}(Y) & =\int_{\tau_{x}} \lim _{Z \rightarrow X} G_{x}(Z, Y) / h_{*}(Z) d \lambda(X) \\
& =\int_{\tau_{x}} \lim _{Z \rightarrow X} G_{x}(Z, Y) / G_{x}(Z, Q) d \lambda^{\prime}(X) .
\end{aligned}
$$

Defining $h_{\mu}$ and $\mu^{\prime}$ in a similar manner, we obtain an equation analogous to (12). Since $h_{\lambda}=h_{\mu}$ by hypothesis, it follows from the uniqueness of the Martin representation for $h_{\lambda}$ in $\Omega_{x}$ that $\lambda^{\prime}=\mu^{\prime}$, whence $\lambda=\mu$.

8.2. The proof of Theorem 1 will now be given. Let $s \in \mathscr{L} \mathscr{D}$ and $w>x>0$. From [15, Theorem 2(i)], the restriction of $s$ to $\partial \Omega_{w}$ is resolutive and $H_{s}^{\Omega_{w}}-s$ is non-negative and superharmonic in $\Omega_{w}$. It follows from the Riesz-Martin decomposition and Theorem B that there is a measure $\lambda_{s}$ on $\tau_{w}$ such that

$$
H_{s}^{\Omega_{w}}(Y)-s(Y)=h(Y)+\int_{\Omega_{w}} G_{w}(X, Y) d \mu_{s}(X)+\int_{\tau_{w}} \lim _{Z \rightarrow X} G_{w}(Z, Y) / h_{*}(Z) d \lambda_{s}(X),
$$

where $h$ is non-negative and harmonic in $\boldsymbol{\Omega}_{w}$ and continuously vanishes on $\tau_{w}$ (see Lemma 6). For any $y<w$, let $Q \in \Omega_{w} \backslash \bar{\Omega}_{y}$ and apply Theorem A to show that there is a positive constant $c$ such that

$$
+\infty>\int_{\Omega_{y} \cup \sigma_{y}} G_{w}(Q, X) d \mu_{s}(X) \geqq c \int_{\Omega_{y} \cup \sigma_{y}} h_{*}(X) d \mu_{s}(X) .
$$

It follows that we can define a measure $v_{s}$ on the Borel subsets $A$ of $\Omega_{w} \cup \tau_{w}$ by

$$
v_{s}(A)=\int_{A \cap \Omega_{w}} h_{*}(Z) d \mu_{s}(Z)+\lambda_{s}\left(A \cap \tau_{w}\right) .
$$


This and (1) enable us to rewrite (13) as

$$
H_{s}^{\Omega_{w}}(Y)-s(Y)=h(Y)+\int_{\Omega_{w} \cup \tau_{w}} h_{*}(Y)\left\{G^{*}(X, Y)-F_{w}(X, Y)\right\} d v_{s}(X) .
$$

Now observe that

$$
\int_{\sigma_{x}} H_{s}^{\Omega} w(Z) d \mu_{x, Y}(Z)=H_{s}^{\Omega} w(Y)-\int_{\tau_{x}} s(Z) d \mu_{x, Y}(Z) .
$$

Also, since $h_{*}(\cdot) F_{w}(X, \cdot)$ is harmonic in $\Omega_{w}$ and continuously vanishes on $\tau_{w}$ (see Lemma 5),

$$
\int_{\sigma_{x}} h_{*}(Z) F_{w}(X, Z) d \mu_{x, Y}(Z)=h_{*}(Y) F_{w}(X, Y) .
$$

It now follows that, if we integrate (15) with respect to harmonic measure on $\sigma_{x}$ (relative to $\Omega_{x}$ ) and use Lemma 5 , we obtain

$$
\begin{gathered}
H_{s}^{\Omega} w(Y)-H_{s}^{\Omega_{x}}(Y)=h(Y)+\int_{\left(W_{w} \backslash W_{x}\right) \cap \bar{\Omega}} h_{*}(Y)\left\{G^{*}(X, Y)-F_{w}(X, Y)\right\} d v_{s}(X) \\
+\int_{W_{x} \cap \bar{\Omega}} h_{*}(Y)\left\{F_{x}(X, Y)-F_{w}(X, Y)\right\} d v_{s}(X) .
\end{gathered}
$$

Subtracting (16) from (15) yields

$$
H_{s}^{\Omega_{x}}(Y)-s(Y)=\int_{W_{x} \cap \bar{\Omega}} h_{*}(Y)\left\{G^{*}(X, Y)-F_{x}(X, Y)\right\} d v_{s}(X),
$$

and so (2) holds for $x<w$ and $\lambda_{s}$ is uniquely (by Lemma 7) defined on $\tau_{w}$. Since $w$ may be arbitrarily large, $\lambda_{s}$ can be defined on all of $\partial \Omega$ (see Lemma 4 (b)).

\section{Proof of Theorem 2}

9.1. We require the following lemma.

Lemma 8. The function

$$
\Phi_{x}(X)=\int_{E} F_{x}(X, Y) d v(Y)
$$

has the constant value $\varphi(x)$ on $\bar{\Omega}_{x}$.

From Lemma $5, \Phi_{x}$ is continuous on $\bar{\Omega}_{x}$ and, if $X \in \sigma_{x}$, then

$$
\Phi_{x}(X)=\int_{E} G^{*}(X, Y) d v(Y)=\Phi(X)=\varphi(x)
$$

(see Lemma 4(d)). Further, by Fubini's theorem and Lemma $5, h_{*} \Phi_{x}$ satisfies the mean-value equality for balls whose closures are contained in $\Omega_{x}$. Thus

$$
h^{*}(\cdot)\left\{\Phi_{x}(\cdot)-\varphi(x)\right\}
$$

is harmonic in $\Omega_{x}$, continuously vanishing on $\partial \Omega_{x}$, and so it is identically zero in $\bar{\Omega}_{x}$. Hence $\Phi_{x}(\cdot)=\varphi(x)$ in $\bar{\Omega}_{x} \cap \Omega$ and so also in $\bar{\Omega}_{x}$ by the continuity of $\Phi_{x}$. 
9.2. To prove Theorem 2, we suppose $x>1$, the case $x<1$ being similar and the case $x=1$ being trivial. Let $v_{s}$ be defined on $W_{x} \cap \bar{\Omega}$ by (14) (with $w=x$ ). Subtracting (17) when $x=1$ from (17) as it stands yields

$$
\begin{gathered}
H_{s}^{\Omega_{x}}(Y)-H_{s}^{\Omega_{1}}(Y)=h_{*}(Y) \int_{\left(W_{x} \backslash W_{1}\right) \cap \bar{\Omega}}\left\{G^{*}(X, Y)-F_{x}(X, Y)\right\} d v_{s}(X) \\
+h_{*}(Y) \int_{W_{1} \cap \bar{\Omega}}\left\{F_{1}(X, Y)-F_{x}(X, Y)\right\} d v_{s}(X),
\end{gathered}
$$

whence, by the joint continuity of $G^{*}, F_{x}$ and $F_{1}$ (see Lemmas 2 and 5),

$$
\begin{gathered}
\mathscr{H}_{s, x}(Y)-\mathscr{I}_{s, 1}(Y)=\int_{\left(W_{x} \backslash W_{1}\right) \cap \bar{\Omega}}\left\{G^{*}(X, Y)-F_{x}(X, Y)\right\} d v_{s}(X) \\
+\int_{W_{1} \cap \bar{\Omega}}\left\{F_{1}(X, Y)-F_{x}(X, Y)\right\} d v_{s}(X)
\end{gathered}
$$

for $Y \in E$. If we now integrate this equation with respect to $v$ and apply Fubini's theorem (recall that the integrands are jointly continuous and non-negative on the range of doublet integration), we obtain

$$
\begin{gathered}
\mathscr{M}(s, x)-\mathcal{N}(s, 1) \\
=\int_{\left(W_{x} \backslash W_{1}\right) \cap \bar{\Omega}}\left\{\Phi(X)-\Phi_{x}(X)\right\} d v_{x}(X)+\int_{W_{1} \cap \bar{\Omega}}\left\{\Phi_{1}(X)-\Phi_{x}(X)\right\} d v_{s}(X) \\
=\int_{\left(W_{x} \backslash W_{1}\right) \cap \bar{\Omega}}\{\Phi(X)-\varphi(x)\} d v_{s}(X)+\{\varphi(1)-\varphi(x)\} v_{s}\left(W_{1} \cap \bar{\Omega}\right),
\end{gathered}
$$

the second equality being a consequence of Lemma 8 .

We now define

$$
\alpha_{s}(t)=v_{s}\left(W_{t} \cap \bar{\Omega}\right) \quad(t \in[1, x]),
$$

which allows (18) to be rewritten as

$$
\mathscr{M}(s, x)-\mathscr{N}(s, 1)=\int_{1}^{x} \varphi(t) d \alpha_{s}(t)-\varphi(x) \alpha_{s}(x)+\varphi(1) \alpha_{s}(1),
$$

since $\varphi$ is continuous and decreasing, and $\alpha_{s}$ is of bounded variation on $[1, x]$. Integrating by parts, this yields

$$
\begin{aligned}
\mathscr{M}(s, x)-\mathcal{N}(s, 1) & =-\int_{1}^{x} \alpha_{s}(t) d \varphi(t) \\
& =-\int_{1}^{x} \lambda_{s}\left(\tau_{t}\right) d \varphi(t)-\int_{1}^{x} \int_{\Omega_{t}} h_{*}(Z) d \mu_{s}(Z) d \varphi(t),
\end{aligned}
$$

and the result follows from the definition of $\mathscr{M}^{*}(s, x)$. 


\section{Proofs of Theorems 3, 4 and 6}

10.1. To prove Theorem 3 , we observe from Theorem 2 that

$$
\mathscr{M}^{*}(s, x)=\mathscr{N}(s, 1)-\int_{1}^{x} \int_{\Omega_{t}} h_{*}(Z) d \mu_{s}(Z) d \varphi(t) .
$$

Since $\varphi$ is decreasing, the double integral is decreasing, and so $\mathscr{M}^{*}(s, x)$ is increasing. Next note that the integrand

$$
\int_{\Omega_{t}} h_{*}(Z) d \mu_{s}(Z)
$$

is right continuous with respect to $\varphi(t)$, so that the double integral in (19) is right differentiable with respect to $\varphi(x)$, and

$$
\frac{d \mathscr{M}^{*}(s, x)}{d \varphi(x)}=-\int_{\Omega_{x}} h_{*}(Z) d \mu_{s}(Z)
$$

holds on $(0,+\infty)$ if the derivative is understood as a right derivative. Since the right hand side of (20) increases as $\varphi(x)$ increases, it follows that $\mathscr{M}^{*}(s, x)$ is convex as a function of $\varphi(x)$ on $(0,+\infty)$, proving (i).

Further, if $s$ is harmonic in $\Omega_{w} \backslash \bar{\Omega}_{y}$, then $\mu_{s}\left(\Omega_{w} \backslash \bar{\Omega}_{y}\right)$ is zero, and it follows from (19) that $\mathscr{M}^{*}(s, x)$ is a linear function of $\varphi(x)$ on $(y, w]$, and so on $[y, w]$ by the continuity of $\mathscr{M}^{*}(s, x)$ on $(0,+\infty)$.

Finally, $\mathscr{M}^{*}(s, x)$ is constant if and only if $\mu_{s}\left(\Omega_{x}\right)$ is zero for all $x$, which is equivalent to $s$ being harmonic in $\Omega$.

10.2. To prove Theorem 4 , we begin by obtaining some inequalities. Let $y>1$ and $Q \in \Omega_{y} \backslash \bar{\Omega}_{1}$. Since $s \in \mathscr{L} \mathscr{D}$, it follows that $s$ has a harmonic majorant in $\Omega_{y}$ (for example, $H_{s}^{\Omega_{y}}$ ) and so, using Theorem A to compare $G(Q, \cdot)$ with $G_{y}(Q, \cdot)$ in $\Omega_{1}$, we have

$$
\int_{\Omega_{1}} G(Q, X) d \mu_{s}(X) \leqq c^{\prime} \int_{\Omega_{1}} G_{y}(Q, X) d \mu_{s}(X)<+\infty .
$$

Let $P \in \Omega_{1}$. Using Theorem $\mathrm{A}$ again, there is a positive constant $c$ such that

$$
c^{-1} h_{*}(Y) / h_{*}(P) \leqq G(X, Y) / G(X, P) \leqq c h_{*}(Y) / h_{*}(P)
$$

for $X \in \Omega \backslash \Omega_{1}$ and $Y \in \Omega_{1 / 2}$, and so, from Lemma 2,

$$
c^{\prime \prime} G(X, P) \leqq h_{*}(X) G^{*}(X, Y) \leqq c^{\prime \prime \prime} G(X, P),
$$

for $X \in \Omega \backslash \Omega_{1}$ and $Y \in \bar{\Omega}_{1 / 2}$, whence

$$
c^{\prime \prime} v(E) G(X, P) \leqq h_{*}(X) \Phi(X) \leqq c^{\prime \prime \prime} v(E) G(X, P)
$$

for $X \in \Omega \backslash \Omega_{1}$. 
We now show that (i) and (iii) are equivalent. The function $s$ has a harmonic majorant in $\Omega$ if and only if $G \mu_{s}$ is a potential in $\Omega$. From (21) this is equivalent to

$$
\int_{\Omega \backslash \Omega_{1}} G(X, P) d \mu_{s}(X)<+\infty,
$$

which, in turn, is equivalent to (iii) because of (22).

It remains to show that (ii) and (iii) are equivalent. Let $x>1$. From (18) and the integration by parts employed at the end of the proof of Theorem 2, we can write

$$
\begin{gathered}
\mathscr{M}^{*}(s, x)=\mathscr{N}(s, 1)+\{\varphi(1)-\varphi(x)\} \int_{\Omega_{1}} h_{*}(X) d \mu_{s}(X) \\
+\int_{\Omega_{x} \backslash \Omega_{1}}\{\Phi(X)-\varphi(x)\} h_{*}(X) d \mu_{s}(X) \\
\leqq \mathscr{N}(s, 1)+\varphi(1) \int_{\Omega_{1}} h_{*}(X) d \mu_{s}(X)+\int_{\Omega_{x} \backslash \Omega_{1}} \Phi(X) h_{*}(X) d \mu_{s}(X) .
\end{gathered}
$$

Thus (iii) implies (ii). On the other hand, the function

$$
\psi(x)=\varphi^{-1}(2 \varphi(x))
$$

is defined for all sufficiently large $x$, and

$$
\int_{\Omega_{x} \backslash \Omega_{1}}\{\Phi(X)-\varphi(x)\} h_{*}(X) d \mu_{s}(X) \geqq \frac{1}{2} \int_{\Omega_{\psi(x)} \backslash \Omega_{1}} \Phi(X) h_{*}(X) d \mu_{s}(X),
$$

since $\Phi(X)>\varphi(\psi(x))$ on $\Omega_{\psi(x)}$. Therefore, from (23),

$$
\mathscr{M}^{*}(s, x) \geqq \mathscr{N}(s, 1)+\frac{1}{2} \int_{\Omega_{\psi(x)} \backslash \Omega_{1}} \Phi(X) h_{*}(X) d \mu_{s}(X),
$$

and so (ii) implies (iii).

10.3. It is now straightforward to deduce Theorem 6 . To show (i), we recall that

$$
\mathscr{M}(s, x)-\mathscr{M}^{*}(s, x)=-\int_{1}^{x} \lambda_{s}\left(\tau_{t}\right) d \varphi(t) .
$$

Since $\varphi$ is decreasing, the right hand side is increasing as a function of $x$. Further, its right derivative with respect to $\varphi(x)$ increases as $\varphi(x)$ increases, so that it is convex with respect to $\varphi(x)$. The result now follows from Theorem 3 (i).

In the case of (ii), $I_{s, x}$ is a harmonic majorant of $s$ in $\Omega_{x}$, and as in Theorem 1, there exists a measure $\lambda_{s}^{\prime}$ on $\partial \Omega$ such that the least harmonic majorant of $s$ in $\Omega_{x}$ is given by

$$
I_{s, x}(Y)-h_{*}(Y) \int_{\tau_{x}}\left\{G^{*}(X, Y)-F_{x}(X, Y)\right\} d \lambda_{s}^{\prime}(X) .
$$

The argument of Theorem 2 now yields that

$$
\mathscr{N}(s, x)=\mathscr{N}(s, 1)-\int_{1}^{x}\left\{\lambda_{s}^{\prime}\left(\tau_{t}\right)+\int_{\Omega_{t}} h_{*}(Z) d \mu_{s}(Z)\right\} d \varphi(t),
$$

and the result follows as in Theorem 3 (i). 


\section{Proof of Theorem 10}

11.1. The following lemma is required.

Lemma 9. Let $0<a<z$ and $S$ be a function which is non-negative and subharmonic in $\Omega_{z} \backslash \bar{\Omega}_{a}$, and vanishes continuously on $\tau_{z} \backslash \tau_{a}$. Then the mean $\mathscr{N}(S, x)$ is real-valued and convex as a function of $\varphi(x)$ for $x \in(a, z)$.

To see this, let $a<b<c<d<y<z$ and define

$$
S_{0}(X)=\left\{\begin{array}{cl}
\mu_{y, X}\left(\tau_{a}\right) & \text { if } X \in \Omega_{y} \\
1 & \text { if } X \in \bar{\tau}_{a} \\
0 & \text { elsewhere in } \bar{\Omega} .
\end{array}\right.
$$

Clearly $S_{0} \in \mathscr{L} \mathscr{D}$, and from Theorem $\mathrm{A}$ there is a positive constant $c^{\prime}$ such that

$$
S(X) \leqq H_{S^{y}}^{\Omega_{y} \backslash \bar{\Omega}_{b}}(X) \leqq c^{\prime} S_{0}(X) \quad\left(X \in \Omega_{d} \backslash \bar{\Omega}_{c}\right),
$$

the first inequality being a consequence of [15, Theorem 2 (i)] and the fact that $\bar{\Omega}_{y} \backslash W_{b}$ is the disjoint union of the closures of finitely many Lipschitz domains (see Definition 1). Hence the function

$$
S^{\prime}(X)=\left\{\begin{array}{cll}
c^{\prime} S_{0}(X) & \text { if } & X \in \bar{\Omega}_{c} \\
\max \left\{c^{\prime} S_{0}(X), S(X)\right\} & \text { if } & X \in\left(W_{z} \cap \bar{\Omega}\right) \backslash \bar{\Omega}_{c}
\end{array}\right.
$$

is subharmonic in $\Omega_{z}$, equal to $S$ in $\Omega_{z} \backslash \bar{\Omega}_{y}$, and satisfies

$$
\limsup _{X \rightarrow Z} S^{\prime}(X)=S^{\prime}(Z) \leqq c^{\prime} \quad\left(Z \in \tau_{z}\right) .
$$

Now suppose that $z>1$. If $x \in(y, z)$, then

$$
\mathscr{M}\left(S^{\prime}, x\right)=\mathscr{N}(S, x)+\mathscr{M}\left(S_{0}, x\right) .
$$

Since $S_{0} \equiv 0$ in $\left(W_{z} \cap \bar{\Omega}\right) \backslash \bar{\Omega}_{y}$, it follows from Theorem 3 (ii) that $\mathscr{M}^{*}\left(S_{0}, x\right)$ is a linear function of $\varphi(x)$ on $(y, z)$. Further, it is easily seen from Lemma 6 and the proof of Theorem 1 that

$$
\lambda_{S_{0}}\left(\tau_{z} \backslash \tau_{y}\right)=0
$$

and so $\mathscr{M}\left(S_{0}, x\right)$ is also a linear function of $\varphi(x)$ on $(y, z)$ (see Definition 2). In addition, Theorem 6 (i) shows that $\mathscr{M}\left(S^{\prime}, x\right)$ is a convex function of $\varphi(x)$ on $(0, z)$ (the fact that $S^{\prime}$ is not defined on all of $\bar{\Omega}$ is immaterial). Hence, from (24), $\mathscr{N}(S, x)$ is a convex function of $\varphi(x)$ on $(y, z)$, and so on $(a, z)$ since $y \in(a, z)$ is arbitrary.

Finally, we point out that, if $z \leqq 1$, then we could define

$$
H_{s, x}(X)=H_{s}^{\Omega x}(X)-\int_{\tau_{z / 2}} s(Z) d \mu_{z / 2, X}(Z)
$$

and corresponding means $\mathscr{M}_{z}(s, x)$ and $\mathscr{M}_{z}^{*}(s, x)$ to avoid the problem of $S^{\prime}$ and $\lambda_{S^{\prime}}$ not being defined on $\tau_{1}$. 
11.2. We now prove Theorem 10. Routine differentiation yields that

$$
\Delta\left\{h_{*} \exp \left(s / h_{*}\right)\right\} \geqq 0
$$

in $\Omega$ if $s \in C^{2}(\Omega)$. If $X \in \Omega$, take a decreasing sequence $\left(s_{m}\right)$ of $C^{2}$ subharmonic functions, and it follows easily that $h_{*} \exp \left(s / h_{*}\right)$ is u.s.c. in $\Omega$ and satisfies the mean-value inequality for balls whose closures are contained in $\Omega$.

Let $z>y>0$. Using the fact that $s \leqq 0$ on $\partial \Omega$ and Theorem A, there is a positive constant $c$ such that

$$
s(X) \leqq I_{s^{+}, z}(X) \leqq c h_{*}(X) \quad\left(X \in \Omega_{y}\right),
$$

whence $h_{*} \exp \left(s / h_{*}\right)$ vanishes continuously on $\tau_{y}$, and so ( $y$ being arbitrary) on all of $\partial \Omega$. It follows from Theorem 6 (i) that

$$
\mathscr{N}\left(h_{*} \exp \left(s / h_{*}\right), x\right)
$$

is increasing as a function of $x$, and so the same is true of $\mathscr{N}_{E}(s, x)$.

Let $0<a<y<w$, and note that (see Lemma 3) the function

where

$$
S=h_{*} \exp \left\{k \Phi+s / h_{*}\right\}
$$

$$
k=\left\{\mathscr{N}_{E}(s, w)-\mathscr{N}_{E}(s, y)\right\} /\{\varphi(y)-\varphi(w)\},
$$

is subharmonic in $\Omega \backslash \bar{\Omega}_{a}$ and vanishes continuously on $\partial \Omega \backslash \tau_{a}$. From Lemma 9, $\mathscr{N}(S, x)$ is real-valued and convex as a function of $\varphi(x)$ on $(a,+\infty)$. Using Lemma 4 (d), if $x \in(y, w)$, then

$$
\begin{aligned}
\exp \{k \varphi(x)\} \exp \left\{\mathscr{N}_{E}(s, x)\right\} \leqq & \left\{\frac{\varphi(x)-\varphi(w)}{\varphi(y)-\varphi(w)}\right\} \exp \{k \varphi(y)\} \exp \left\{\mathscr{N}_{E}(s, y)\right\} \\
& +\left\{\frac{\varphi(y)-\varphi(x)}{\varphi(y)-\varphi(w)}\right\} \exp \{k \varphi(w)\} \exp \left\{\mathscr{N}_{E}(s, w)\right\}
\end{aligned}
$$

which, upon rearranging, using (25) and taking logs, yields

as required.

$$
\mathscr{N}_{E}(s, x) \leqq\left\{\frac{\varphi(x)-\varphi(w)}{\varphi(y)-\varphi(w)}\right\} \mathscr{N}_{E}(s, y)+\left\{\frac{\varphi(y)-\varphi(x)}{\varphi(y)-\varphi(w)}\right\} \mathscr{N}_{E}(s, w)
$$

\section{Applications to the whole space}

In this and subsequent sections, when $(n-1)$-dimensional surface area measure on the boundary of a domain exists, it will be denoted by $\sigma$. Thus, in particular, the spherical mean of a suitably defined function $f$ is given by

$$
\mathscr{L}(f: X, r)=c_{n}^{-1} r^{1-n} \int_{\partial B(X, r)} f(Z) d \sigma(Z),
$$

where $c_{n}$ denotes the surface area of $\partial B(O, 1)$. 
Let $\Omega=\mathbf{R}^{n}(n \geqq 3)$ and $h_{*} \equiv 1$. First consider $E=\{O\}$, and $v$ to be the Dirac measure at the origin. Clearly $\Phi(X)=|X|^{2-n}$ so that $x=+\infty$ and, if we take $\varphi(x)=x^{2-n}$, then $\Omega_{x}=B(O, x)$ for all $x$, and

$$
\mathscr{M}^{*}(s, x)=\mathscr{M}(s, x)=\mathscr{N}(s, x)=H_{s}^{B(o, x)}(O)=\mathscr{L}(s: O, x) .
$$

The following well-known results are now seen to be special cases of the results in Sections 4 and 5.

Theorem 11. Let $s$ be subharmonic in $\mathbf{R}^{n}$ ( $n \geqq 3$ ) and $u$ be positive and superharmonic. Then

(i) $\mathscr{L}(s: O, r)$ is convex as a function of $r^{2-n}$ and increasing as a function of $r$;

(ii) if $R_{2}>R_{1}>0$ and $s$ is harmonic in $B\left(O, R_{2}\right) \backslash \bar{B}\left(O, R_{1}\right)$, then $\mathscr{L}(s: O, r)$ is a linear function of $r^{2-n}$ on $\left[R_{1}, R_{2}\right]$;

(iii) $s$ has a harmonic majorant in $\mathbf{R}^{n}$ if and only if $\mathscr{L}(s: O, r)$ is bounded above for $r>0$, which in turn is equivalent to

$$
\int_{\mathbf{R}^{n}}(1+|X|)^{2-n} d \mu_{s}(X)<+\infty ;
$$

(iv) the expressions

and

$$
\sup \{s(X):|X|=r\}
$$

$$
\log \mathscr{L}(\exp s: O, r)
$$

are convex as functions of $r^{2-n}$ and increasing as functions of $r>0$;

(v) if $s \geqq 0$ and $p \geqq 1$, then the same is true of

$$
\left\{\mathscr{L}\left(s^{p}: O, r\right)\right\}^{1 / p} ;
$$

(vi) if $p \in(-\infty, 0) \cup(0,1)$, then

$$
\left\{\mathscr{L}\left(u^{p}: O, r\right)\right\}^{1 / p}
$$

is concave as a function of $r^{2-n}$ and decreasing as a function of $r>0$.

It is natural to ask what results could be obtained for different choices of $E$ and $v$. The simplest cases to consider would be when $E$ is an $m$-dimensional ball, where $0<m \leqq n-1$, and $v$ is symmetrically distributed on $E$. In order to simplify the discussion, we shall restrict ourselves to the case $\Omega=\mathbf{R}^{3}$, and again let $h_{*} \equiv 1$.

Example 3. (i) Fix $c>0$ and let

$$
E=\left\{X \in \mathbf{R}^{3}: x_{1}=x_{2}=0 \text { and }\left|x_{3}\right| \leqq c\right\} .
$$

It will be convenient to work in prolate spheroidal polar co-ordinates, so that

$$
\begin{aligned}
& x_{1}=c \sinh \eta \sin \theta \cos \psi, \\
& x_{2}=c \sinh \eta \sin \theta \sin \psi, \\
& x_{3}=c \cosh \eta \cos \theta,
\end{aligned}
$$


where

$$
0 \leqq \eta<+\infty, \quad 0 \leqq \theta \leqq \pi, \quad 0 \leqq \psi<2 \pi .
$$

Choosing $\varphi(x)=\log \operatorname{coth}\left(\frac{1}{2} x\right)$, it is routine to deduce that $\Omega_{x}$ is the region bounded by the prolate spheroid

and that

$$
x_{3}^{2} / \cosh ^{2} x+\left(x_{1}^{2}+x_{2}^{2}\right) / \sinh ^{2} x=c^{2},
$$

$$
\mathscr{M}(s, x)=\mathscr{N}(s, x)=(4 \pi)^{-1} c \int_{0}^{\pi} \int_{0}^{2 \pi} s(x, \theta, \psi) \sin \theta d \psi d \theta .
$$

A theorem analogous to Theorem 11 can now be written down for the prolate spheroidal mean $\mathscr{M}(s, x)$; convexity is in terms of $\log \operatorname{coth}\left(\frac{1}{2} x\right)$.

(ii) If similar calculations are performed for

$$
E=\left\{X \in \mathbf{R}^{3}: x_{1}^{2}+x_{2}^{2} \leqq c^{2} \text { and } x_{3}=0\right\},
$$

analogous results for an oblate spheroidal mean are obtained. Details are left to the reader.

\section{Applications to the half-space}

Let $\Omega=\mathbf{R}^{n-1} \times(0,+\infty)(n \geqq 2)$, let $E=\{O\}$ and $v$ be the mass $c_{n} /(2 n)$ at $O$, and $h_{*}(X)=x_{n}$ in $\Omega$. From [25, Lemma 1],

$$
2 \gamma_{n}^{-1} c_{n}^{-1} x_{n} y_{n}\left|\left(X^{\prime},-x_{n}\right)-Y\right|^{-n} \leqq G(X, Y) \leqq 2 \gamma_{n}^{-1} c_{n}^{-1} x_{n} y_{n}|X-Y|^{-n},
$$

where $\gamma_{n}$ is as defined in Section 3. Hence

$$
\Phi(X)=c_{n}(2 n)^{-1} G^{*}(X, O)=\gamma_{n}^{-1} n^{-1}|X|^{-n} .
$$

Thus $x=+\infty$ and, defining $\varphi(x)=\gamma_{n}^{-1} n^{-1} x^{-n}$ for $x \in(0,+\infty)$, it follows that $\Omega_{x}=B(O, x) \cap \Omega$. It now follows from [14, Section 8$]$ that

and

$$
\mathscr{N}(s, x)=x^{-n-1} \int_{\sigma_{x}} y_{n} s(Y) d \sigma(Y),
$$

$$
\mathscr{M}(s, x)=\mathscr{N}(s, x)+\int_{1}^{x} t^{-n-1} \int_{\tau_{t}} s(Y) d \sigma(Y) d t
$$

In this context, Theorems $2-6$ are improvements of the main results of [4].

\section{Applications to the infinite cylinder}

Instead of deducing known results concerning the infinite strip, [5], and infinite cone, [13], we follow the pattern of [14] and derive previously unpublished results for the infinite cylinder.

Let $\Omega=\left\{\left(X^{\prime}, x_{n}\right):\left|X^{\prime}\right|<1\right\}, \quad(n \geqq 2)$. We shall employ the Bessel function $J_{(n-3) / 2}$ defined in Watson [28, pp. 40-42], the least positive zero of which will be 
denoted by $a_{n}$. We write

and

$$
\psi(t)=t^{(3-n) / 2} J_{(n-3) / 2}\left(a_{n} t\right) \quad(t>0)
$$

$$
b_{n}=a_{n} J_{(n-1) / 2}\left(a_{n}\right)>0,
$$

(see [28, p. $45(4)$ and p. $479 \S 15.22]$ ). Recalling (see [14, Lemma 3]) that the functions $\psi\left(\left|X^{\prime}\right|\right) \exp \left( \pm a_{n} x_{n}\right)$ are positive and harmonic in $\Omega$ and vanish on $\partial \Omega$, we can define

and

$$
\begin{gathered}
\left.E=\left(X^{\prime}, x_{n}\right):\left|X^{\prime}\right|<1, x_{n}=0\right\}, \\
d v(X)=2\left\{\psi\left(\left|X^{\prime}\right|\right)\right\}^{2} d X^{\prime} d \delta_{0}\left(x_{n}\right) \quad(X \in E),
\end{gathered}
$$

$$
h_{*}(X)=\psi\left(\left|X^{\prime}\right|\right) \cosh \left(a_{n} x_{n}\right),
$$

where $\delta_{0}$ denotes the Dirac measure at the origin of $\mathbf{R}$.

Next we determine $\Phi$, and hence $\Omega_{x}$. Clearly the function

$$
v(X)=a_{n}^{-1} \exp \left(-a_{n}\left|x_{n}\right|\right) \psi\left(\left|X^{\prime}\right|\right) \quad(X \in \Omega)
$$

is positive and superharmonic in $\Omega$, harmonic in $\Omega \backslash E$, bounded above on $\bar{\Omega}$, and continuously vanishing on $\partial \Omega$. From a result of Bouligand [18, Corollary 9.20], the greatest harmonic minorant of $v$ in $\Omega$ is zero, and so $v$ is the potential whose measure is given by $\mu=-\gamma_{n} \Delta v$. If we now let $\Psi$ be a $C^{\infty}$ function with compact support in $\Omega$, it follows from Green's theorem (as in [14, Section 9]) that

whence

$$
(\Delta v)(\psi)=-2 \int_{\left\{\left|X^{\prime}\right|<1\right\}} \Psi\left(X^{\prime}, 0\right)\left|X^{\prime}\right|^{(3-n) / 2} J_{(n-3) / 2}\left(a_{n}\left|X^{\prime}\right|\right) d X^{\prime},
$$

and so

$$
d \mu(X)=2 \gamma_{n}\left|X^{\prime}\right|^{(3-n) / 2} J_{(n-3) / 2}\left(a_{n}\left|X^{\prime}\right|\right) d X^{\prime} d \delta_{0}\left(x_{n}\right)
$$

$$
\gamma_{n}^{-1} v(X)=\int_{E} G(X, Y) / h_{*}(Y) d v(Y)=h_{*}(X) \Phi(X) .
$$

Hence, dividing through by $h_{*}(X)$,

$$
\begin{aligned}
\Phi(X) & =\gamma_{n}^{-1} a_{n}^{-1} \exp \left(-a_{n}\left|x_{n}\right|\right) \operatorname{sech}\left(a_{n} x_{n}\right), \\
& =\gamma_{n}^{-1} a_{n}^{-1}\left\{1-\tanh \left(a_{n}\left|x_{n}\right|\right)\right\},
\end{aligned}
$$

and so $\varkappa=\gamma_{n}^{-1} a_{n}^{-1}$. If we define

it follows that

$$
\varphi(x)=\gamma_{n}^{-1} a_{n}^{-1}\left\{1-\tanh \left(a_{n} x\right)\right\},
$$

$$
\Omega_{x}=\left\{X \in \Omega:\left|x_{n}\right|<x\right\}
$$

and so, from [14, Section 9],

$$
\mathscr{N}(s, x)=\operatorname{sech}\left(a_{n} x\right) \int_{\sigma_{x}} \psi\left(\left|X^{\prime}\right|\right) s(X) d \sigma(X),
$$


and

$$
\mathscr{M}(s, x)=\mathscr{N}(s, x)+b_{n} \int_{1}^{x} \operatorname{sech}^{2}\left(a_{n} t\right) \int_{\tau_{t}} s(X) \cosh \left(a_{n} x_{n}\right) d \sigma(X) d t
$$

The results of Sections 4 and 5 may now be applied to subharmonic functions in the infinite cylinder, and convexity as a function of $\varphi(x)$ can clearly be equivalently stated as convexity as a function of $\tanh \left(a_{n} x\right)$.

\section{References}

[1] Ahlfors, L. V.: On Phragmén-Lindelöf's principle. - Trans. Amer. Math. Soc. 41, 1937, $1-8$.

[2] AHLfors, L. V.: Remarks on Carleman's formula for functions in a half-plane. - SIAM J. Numer. Anal. 3, 1966, 183-187.

[3] Armitage, D. H.: Half-spherical means and harmonic majorization in half-spaces. - J. London Math. Soc. (2) 19, 1979, 457-464.

[4] Armitage, D. H.: A Nevanlinna theorem for superharmonic functions in half-spaces, with applications. - J. London Math. Soc. (2) 23, 1981, 137-157.

[5] Armitage, D. H., and T. B. Fugard: Subharmonic functions in strips. - J. Math. Anal. Appl. 89, 1982, 1-27.

[6] Armitage, D. H., and S. J. Gardiner: Some Phragmén-Lindelöf and harmonic majorization theorems for subharmonic functions. - J. Math. Anal. Appl. 102, 1984, 156-174.

[7] Brawn, F. T.: The Green and Poisson kernels for the strip $\left.\mathbf{R}^{n} \times\right]$ 0, 1[. - J. London Math. Soc. (2) 2, 1970, 439-454.

[8] Brawn, F. T.: Mean value and Phragmén-Lindelöf theorems for subharmonic functions in strips. - J. London Math. Soc. (2) 3, 1971, 689-698.

[9] Brawn, F. T.: Positive harmonic majorization of subharmonic functions in strips. - Proc. London Math. Soc. (3) 27, 1973, 261-289.

[10] DAhlberG, B. E. J.: Estimates of harmonic measure. - Arch. Rational Mech. Anal. 65, 1977, $275-288$.

[11] Dinghas, A.: Über einige Konvexitätsätze für die Mittelwerte von subharmonischen Funktionen. - J. Math. Pures Appl. 44, 1965, 223-247.

[12] Fugard, T. B.: Growth and convexity properties of harmonic and subharmonic functions. M. Sc. Thesis, The Queen's University of Belfast, 1979.

[13] Fugard, T. B.: Harmonic and subharmonic functions in cones and half-spaces. - Ph. D. Thesis, The Queen's University of Belfast, 1981.

[14] Gardiner, S. J.: Harmonic majorization of subharmonic functions in unbounded domains. Ann. Acad. Sci. Fenn. Ser. A I Math. 8, 1983, 43-54.

[15] GARDINER, S. J.: Local and global majorization of subharmonic functions. - J. Analyse Math. $42,1983,175-184$.

[16] Hayman, W. K., and P. B. Kennedy: Subharmonic functions, Vol. I. - London Mathematical Society Monographs, No. 9, Academic Press, London-New York-San Francisco, 1976.

[17] HeIns, M.: On some theorems associated with the Phragmén-Lindelöf principle - Ann. Acad. Sci. Fenn. Ser. A I Math. 46, 1948, 1-10.

[18] HeLms, L. L.: Introduction to potential theory. - Wiley-Interscience, a division of John Wiley \& Sons, New York-London-Sydney-Toronto, 1969.

[19] Huber, A.: On functions subharmonic in a half-space. - Trans. Amer. Math. Soc. 82, 1956, $147-159$. 
[20] Hunt, R. A., and R. L. WheEden: Positive harmonic functions on Lipschitz domains. - Trans. Amer. Math. Soc. 147, 1970, 507-527.

[21] Jerison, D. S., and C. E. KeNIG: Boundary behavior of harmonic functions in non-tangentially accessible domains. - Adv. in Math. 46, 1982, 80-147.

[22] Kuran, Ü.: Study of superharmonic functions in $\mathbf{R}^{n} \times(0,+\infty)$ by a passage to $\mathbf{R}^{n+3}$. - Proc. London Math. Soc. (3) 20, 1970, 276-302.

[23] KURAN, Ü.: On half-spherical means of subharmonic functions in half-spaces. - J. London Math. Soc. (2) 2, 1970, 305-317.

[24] NÄ̈M, L.: Sur le rôle de la frontière de R. S. Martin dans la théorie du potentiel. - Ann. Inst. Fourier (Grenoble) 7, 1957, 183-281.

[25] Nualtaranee, S.: On least harmonic majorants in half-spaces. - Proc. London Math. Soc. (3) $27,1973,243-260$.

[26. RIESz, F.: Sur les fonctions subharmoniques et leur rapport à la théorie du potentiel. - Acta Math. 48, 1926, 329-343.

[27] TsuJI, M.: On a positive harmonic function in a half-plane. - Japan J. Math. 15, 1939, 277285.

[28] Watson, G. N.: A treatise on the theory of Bessel functions (2nd edition). - Cambridge University Press, London, 1944.

[29] Wu, J.-M. G.: Convexity of integral means of subharmonic functions. - Proc. Amer. Math. Soc. 60, 1976, 225-230.

[30] Wu, J.-M. G.: Comparisons of kernel functions, boundary Harnack principle and relative Fatou theorem on Lipschitz domains. - Ann. Inst. Fourier (Grenoble) 28, 1978, 147-167.

Mrs. Marjatta Lappalainen

14 January 1986

The Queen's University of Belfast

Department of Pure Mathematics

Belfast BT7 1NN

Northern Ireland

Present adress

University College

Department of Mathematics

Belfield, Dublin 4

Republic of Ireland

Received 9 February 1984 